\title{
Will the child with mania please stand up? ${ }^{\dagger}$
}

\author{
Gabrielle A. Carlson
}

\section{Summary}

Rates of bipolar disorder in any sample will depend on the sample studied, the interview used, with whom, how the information is integrated, the criteria applied, and the conceptualisation of a manic episode and its symptoms. A cross-national diagnostic study is needed to determine the implications of different approaches and definitions.

\section{Declaration of interest}

G.A.C. receives research funding from GlaxoSmithKline and the National Institute for Mental Health and has consulted to Eli Lilly. She is on the scientific advisory boards of the Child and Adolescent Bipolar Foundation and Children and Adults with Attention-Deficit/Hyperactivity Disorder (CHADD) for Suffolk County, New York, USA.
Gabrielle Carlson is Professor of Psychiatry and Paediatrics and Director of Child and Adolescent Psychiatry at Stony Brook University School of Medicine in the USA. She has researched the subject of children and adults with bipolar disorder for over 35 years.

The frequency of type 1 bipolar disorder in children and its overlap with attention-deficit hyperactivity disorder (ADHD) have long been a source of controversy in the USA and more recently elsewhere. ${ }^{1,2}$ The paper by Hassan et al allows us to examine some of the assessment, sampling and conceptual differences between studies that contribute to these large differences in rates. ${ }^{3}$ In their study, a clinical sample of children from the UK (mean age 11 years) was recruited for a genetic study of ADHD. Diagnoses were made using the parent version (and child version for older children) of the Child and Adolescent Psychiatric Assessment (CAPA) research diagnostic interview. ${ }^{4}$ Teacher information was also obtained for ADHD symptoms. Only one child with ADHD (0.5\% of the sample) met DSM-IV criteria for hypomania and none met the criteria for mania. This contrasts with rates that range from $2 \%$ to $23 \%$ for mania/mixed mania in the USA, ${ }^{5,6}$ and $10 \%$ in an Italian sample. ${ }^{7}$ What accounts for such disparate findings?

\section{Assessment and sampling issues}

Although standardised instruments should increase diagnostic accuracy and consistency, the interviews used to ascertain diagnoses in samples with high rates of bipolar disorder and in this study are quite different. The CAPA is a semi-structured, interviewer-based instrument that asks about expansive mood 'intrusive and uncontrollable in almost all activities'; irritable mood must be present within the same $24 \mathrm{~h}$ period as expansive mood. Other mania symptoms that accompany expansive/irritable mood must represent a change in behaviour. Severity is rated. Most importantly, the CAPA ascertains symptoms only for the previous 3 months.

The childhood version of the Schedule for Affective Disorders and Schizophrenia (K-SADS), the interview used most commonly in the US bipolar disorder studies, has several iterations. The 1987 epidemiologic version, KSADS-E, ${ }^{8}$ is virtually respondent-based with a 'yes/no' format. The interviewer asks screening questions: 'Did you ever have a time when you felt very good, or too cheerful or high, terrific or great? Did it ever get you into trouble or have people worry about you?' 'Did you ever have a period when you were easily annoyed, irritable, or got angry for little reason?' If

†See pp. 195-198, this issue. the response to either item is positive, other symptoms of mania are sought. The two moods do not have to co-occur, nor must the other manic symptoms be part of the same mood period, and there is no specific instruction that overlapping ADHD symptoms intensify during an episode. This version gives a lifetime and current diagnosis, not a 3-month diagnosis. More recent versions of the K-SADS rate severity but still make current and lifetime diagnoses. ${ }^{9}$ The Washington University K-SADS ascertains symptoms, not episode onset and offset. ${ }^{10}$ The similarities and differences in samples assessed with these specific interviews have not been examined but should be. In a comparison of the K-SADS and another interview, my colleagues and I found that rates of internalising disorders were most sensitive to interview type, and obtaining information only from parents inflated these rates compared with a best-estimate diagnostic procedure. ${ }^{11}$ Also relevant in making diagnoses is whether raters use information from any informant (the 'or' rule) or require a consensus between parent and youth (the 'and' rule). In the USA, most people use the or rule. It is not clear what Hassan et al did. The UK assessment included teacher input; US studies almost never do.

How similar are the samples finding high and low rates of bipolar disorder? Biederman's US samples of children with ADHD were similar to Hassan et al's in rates of oppositional defiant disorder (48-56\% v. 42\% in the Hassan et al sample) and conduct disorder (10-15\% in the former $v .14 .5 \%$ in the latter). ${ }^{12}$ However, the US children were significantly moodier. Rates of anxiety (about 25\%) and major depression (38\%) were much higher than in the Hassan et al sample (2\% and $1.5 \%$ respectively). This again may be the result of a lifetime $v$. 3-month issue which is less important in externalising disorders and more problematic for episodic mood disorders.

\section{Conceptualisation}

The largest contribution to rate differences probably results from how differently clinicians conceptualise mania/bipolar disorder and its symptoms and apply criteria. Dubicka et al examined differences in the ways clinicians from the UK and the USA classified vignettes of children with a variety of conditions. ${ }^{13}$ The hypothesis was that rates of mania would be much higher in the USA than in the UK, and that was indeed what was found. In the case designed to illustrate the diagnostic conundrum between mania and hyperactivity with oppositional/irritable and moody behaviour, three-quarters of US clinicians and only a third of UK clinicians rated the child as having mania. Most UK clinicians felt the child had hyperkinetic conduct disorder. Dubicka et al also observed that DSM and ICD criteria for bipolar disorder differ. ${ }^{13}$ Episodes defined as differences in patient functioning with return to 
premorbid state are more clearly defined in ICD criteria than in the DSM. In fact, the DSM-IV episode criterion for mania is so ambiguous that it is likely to be changed for DSM-5. ${ }^{14}$ Interviewers who lack experience evaluating and treating true mania, and rely only on patients' responses to questions, probably rate mania differently from those who use pattern recognition. It appeared in the study that pattern recognition more often motivated diagnosis in the UK than in the USA. In addition, clinicians in the UK recognised the specific symptoms meant to illustrate mania, but interpreted them differently from US clinicians.

Clearly, the Hassan et al study adds to the 'bipolar controversy'. The controversy has arisen for a number of reasons that go beyond the question of ADHD and mania. ${ }^{1}$ The controversy is whether a broader definition of mania with less clear-cut episodes and more concurrent comorbidity represents a developmentally altered condition which will change in adulthood, or a condition which, because of its earlier onset, has a worse prognosis - i.e. the childhood version of adult mixed and rapid cycling bipolar disorder $v$. classic bipolar disorder, or a stable, temperamental, genetically related construct that may or may not predict bipolar disorder, in the same way that schizotypal personality is disabling in itself but is only sometimes related to schizophrenia.

Some would suggest that the major difference between ADHD and mania is the presence of euphoria and grandiosity. The 'bipolar controversy' is about whether one can interpret euphoria and grandiosity in a young child in the same way one does in an adult. How does one ask a child about a concept as sophisticated as euphoria? Should the mood be disengaged from context? Goofy and silly children are not necessarily euphoric - witness the 'class clown' who has ADHD. Grandiosity requires that a child understand the reality of what is possible. Interviewers ask the child, 'Have you ever felt you were the smartest kid in your class, or the best baseball player ever, or are you super self-confident? Or do you have special plans for the world?' Are children grandiose because they think they will be the next rock star or home-run champion? A little probing reveals that these children wish that they had such attributes, but do not really think that they do. Not only have these attempts at 'developmentally altering' criteria not been tested, interviewers probably probe, apply criteria and interpret responses differently, creating bipolar disorder samples that are not the same even within the USA, let alone between the USA and other countries.

A major question is whether chronically irritability, mood dysregulation or rages represent bipolar disorder. ${ }^{15}$ Although bipolar disorder is more 'biological', and may feel less pejorative than oppositional defiant or conduct disorders (previous labels for irritable, aggressive children), bipolar disorder has not been the outcome, at least in community samples of irritable, oppositional children. ${ }^{16}$

\section{Recommendation}

The prevalence of bipolar disorder varies even in adults, as does age at onset. ${ }^{17}$ I submit, however, that the way in which the methodological issues outlined above are addressed will determine which child is given a diagnosis of mania in any given sample, past or present. In order to emerge from the controversy we need a study similar to the cross-national International Pilot Study of Schizophrenia, ${ }^{18}$ launched 50 years ago because of very different rates of schizophrenia and manic depression found in the USA compared with the UK. This landmark study used actual patients observed by clinicians from both countries and revealed important reasons for discrepant rates. This study gave rise to the assessment and nosological revolution that has radically changed both DSM and ICD. The issue of bipolar disorder in children needs the same approach, with different diagnostic camps within the USA and internationally taking part. Until we understand how clinicians ask and understand parent and child responses to questions about their episodes and symptoms, and how they apply criteria that the DSM and ICD committees establish, we are trapped in an endless nosological debate.

Gabrielle A. Carlson, MD, Professor, Department of Child and Adolescent Psychiatry, Stony Brook University School of Medicine, Putnam Hall - South Campus, Stony Brook, New York 11794-8790, USA. E-mail: Gabrielle.Carlson@StonyBrook.edu

First received 29 Jul 2010, final revision 15 Nov 2010, accepted 17 Nov 2010

\section{References}

1 Carlson GA. Child and adolescent mania - diagnostic considerations. J Child Psychol Psychiatry 1990; 31: 331-41.

2 Soutullo CA, Chang KD, Díez-Suárez A, Figueroa-Quintana A, EscamillaCanales I, Rapado-Castro $\mathrm{M}$, et al. Bipolar disorder in children and adolescents: international perspective on epidemiology and phenomenology. Bipolar Disord 2005; 7: 497-506.

3 Hassan A, Agha SS, Langley K, Thapar A. Prevalence of bipolar disorder in children and adolescents with attention-deficit hyperactivity disorder. Br J Psychiatry 2011; 198: 195-8.

4 Angold A, Costello EJ. The Child and Adolescent Psychiatric Assessment (CAPA). J Am Acad Child Adolesc Psychiatry 2000; 39: 39-48.

5 McGough JJ, Loo SK, McCracken JT, Dang J, Clark S, Nelson SF, et al., CBCL pediatric bipolar disorder profile and ADHD: comorbidity and quantitative trait loci analysis. J Am Acad Child Adolesc Psychiatry 2008; 47: 1151-7.

6 Biederman J, Faraone S, Mick E, Wozniak J, Chen L, Ourlette C, et al. Attention-deficit hyperactivity disorder and juvenile mania: an overlooked comorbidity? J Am Acad Child Adolesc Psychiatry 1996; 35: 997-1008.

7 Donfrancesco R, Miano S, Martines F, Ferrante L, Melegari MG, Masi G. Bipolar disorder co-morbidity in children with attention deficit hyperactivity disorder. Psychiatry Res 2010; Aug 5 (Epub ahead of print).

8 Orvaschel H, Puig-Antich J. Schedule for Affective Disorders and Schizophrenia for School-Age Children: Epidemiologic Version. Nova University, 1987.

9 Kaufman J, Birmaher B, Brent D, Rao U, Flynn C, Moreci P, et al. Schedule for Affective Disorders and Schizophrenia for School-Age Children - Present and Lifetime version (K-SADS-PL): initial reliability and validity data. J Am Acad Child Adolesc Psychiatry 1997; 36: 980-8.

10 Geller B, Zimerman B, Williams M, Bolhofner K, Craney JL, DelBello MP, et al. Reliability of the Washington University in St Louis Kiddie Schedule for Affective Disorders and Schizophrenia (WASH-U-KSADS) mania and rapid cycling sections. J Am Acad Child Adolesc Psychiatry 2001; 40: 450-5.

11 Carlson GA, Kashani JH, de Fatima Thomas M, Vaidya A, Daniel AE. Comparison of two structured interviews on a psychiatrically hospitalized population of children. J Am Acad Child Adolesc Psychiatry 1987; 26: 645-8.

12 Biederman J, Faraone SV, Wozniak J, Mick E, Kwon A, Aleardi M. Further evidence of unique developmental phenotypic correlates of pediatric bipolar disorder: findings from a large sample of clinically referred preadolescent children assessed over the last 7 years. J Affect Disord 2004; 82 (suppl 1): S45-58.

13 Dubicka B, Carlson GA, Vail A, Harrington R. Prepubertal mania: diagnostic differences between US and UK clinicians. Eur Child Adolesc Psychiatry 2008; 17: 153-61.

14 American Psychiatric Association. Issues Pertinent to a Developmental Approach to Bipolar Disorder. American Psychiatric Association, 2010 (http://www.dsm5.org/Proposed\%20Revision\%20Attachments/ APA\%20Developmental\%20Approaches\%20to\%20Bipolar\%20Disorder.pdf).

15 Leibenluft E. Severe mood dysregulation, irritability, and the diagnostic boundaries of bipolar disorder in youth. Am J Psychiatry 2010; Dec 1 (Epub ahead of print).

16 Stringaris A, Goodman R. Longitudinal outcome of youth oppositionality: irritable, headstrong, and hurtful behaviors have distinctive predictions. J Am Acad Child Adolesc Psychiatry 2009; 48: 404-12.

17 Hodgins S, Faucher $B$, Zarac A, Ellenbogen M. Children of parents with bipolar disorder. A population at high risk for major affective disorders. Child Adolesc Psychiatr Clin N Am 2002; 11: 533-53.

18 Cooper JE, Kendall RE, Gurland BJ, Sharpe L, Copeland JRM, Simon R. Psychiatric Diagnosis in New York and London: A Comparative Study of Mental Hospital Admissions. Maudsley Monographs 20. Institute of Psychiatry, 1972. 